\title{
The developing human brain: a frontiers research topic
}

\section{Silvia A. Bunge*}

Department of Psychology and Helen Wills Neuroscience Institute, University of California Berkeley, Berkeley, CA, USA

${ }^{*}$ Correspondence: sbunge@berkeley.edu

\section{INTRODUCTION}

The field of developmental cognitive neuroscience (DCN) examines how the human brain and behavior change over the lifespan, in particular over infancy, childhood, and adolescence. DCN researchers conduct basic research on typical brain development, and/or research on pediatric populations with neurodevelopmental disorders.

Developmental cognitive neuroscience is not child's play - it is a complex and challenging, but exciting, area of research. It is highly multidisciplinary, in that it builds on the fields of developmental, cognitive, affective/social, and clinical psychology and neuroscience. DCN research also relies on advances in computer science, physics, and mathematics. Increasingly, as we learn more about the developing human brain, findings from DCN have strong implications for medicine, education, law, public health, and social welfare.

The story of this compendium begins with a highly successful conference on neurocognitive development held at UC Berkeley in 2009 - a 3-day event with over 200 attendees and over 60 scientific presentations from around the United States and Europe. I thank Brian Meyer, a graduate student in Psychology at UC Berkeley, for helping me to organize this conference. Following on the heels of this conference, postdoctoral fellow Dr. Elizabeth O'Hare and I solicited submissions to a special issue of Frontiers in Human Neuroscience focused on the developing human brain. Dr. O'Hare deserves my gratitude for helping to handle the peer review of these submissions.

This Research Topic includes a selection of empirical and review papers from top DCN researchers, organized into six interrelated sections.

Section I examines how our visual and auditory systems develop to support the perception of complex stimuli. Recent DCN research highlights the point that, contrary to common belief, perceptual development is not complete after the first few years of life.

Face processing - both the recognition of individuals and of specific emotions - is a particularly challenging problem for the visual system, and continues to improve through adolescence. Section II extends the theme of Section I by exploring in more detail the perception of faces, which is critical for social functioning. This section ends with the exploration of deficits in social functioning after pediatric brain injury, which serves as a bridge from studies of perception to studies of action.

Section III examines two key neural changes that underlie shifts in behavior over development. The first is a change in the processing of rewards and feedback, which are powerful motivators of behavior. The second is a change in the ability to control behavioral responses, known as cognitive control or executive function, or more generally as self-regulation.

Developmental cognitive neuroscience researchers have begun to use sophisticated network analysis tools to examine developmental changes in the strength of interactions between brain regions in large-scale networks. Section IV explores how these brain networks are influenced by common genetic variation or by the presence of a disorder with a strong hereditary component.

Section $V$ features a relatively new area of research within DCN: the exploration of societal influences on brain development. Two of the papers in this section compare neurocognitive function in children from North American and Chinese families. The other two papers review what is known currently about the influences of childhood adversity and socioeconomic status on brain development.

Finally, Section VI showcases several new ways of thinking about, and studying, the developing human brain. The first paper is an opinion piece highlighting the relevance to $\mathrm{DCN}$ of research at the level of neural circuits. The second paper shows how we can go beyond conventional fMRI imaging analyses to examine changes in brain function at a finer grain using multivariate pattern classification algorithms.

In conclusion, this compendium shows us both where the field of DCN is currently, and where it is headed. The advances over the last few years have been breathtaking - and the best is yet to come.

Received: 23 February 2012; accepted: 23 February 2012; published online: 12 March 2012.

Citation: Bunge SA (2012) The developing human brain: a frontiers research topic. Front. Hum. Neurosci. 6:47. doi: 10.3389/fnhum.2012.00047

Copyright $\odot 2012$ Bunge. This is an open-access article distributed under the terms of the Creative Commons Attribution Non Commercial License, which permits non-commercial use, distribution, and reproduction in other forums, provided the original authors and source are credited. 